\title{
THE ASYMPTOTIC BEHAVIOR OF FIRMLY NONEXPANSIVE MAPPINGS
}

\author{
SIMEON REICH AND ITAI SHAFRIR
}

\begin{abstract}
We present several new results on the asymptotic behavior of firmly nonexpansive mappings in Banach spaces and in the Hilbert ball.
\end{abstract}

Let $D$ be a subset of a (real) Banach space $X$. Recall that a mapping $T: D \rightarrow X$ is said to be firmly nonexpansive $[2,4]$ if for each $x$ and $y$ in $D$, the convex function $f:[0,1] \rightarrow[0, \infty)$ defined by

$$
f(s)=|(1-s) x+s T x-((1-s) y+s T y)|
$$

is nonincreasing. Note that $T$ is firmly nonexpansive if and only if it is the resolvent $(I+A)^{-1}$ for some accretive operator $A \subset X \times X$, and that any linear projection of norm 1 is firmly nonexpansive. It is known [5] that firmly nonexpansive mappings have several remarkable properties which are not shared by all nonexpansive mappings. Our purpose here is to present several new results on the asymptotic behavior of these mappings.

We solve, in particular, a problem which was left open in [5] (Corollary 1), use a new geometric property of Banach spaces (Theorem 2), and present Hilbert ball analogues of some of our results (Theorem 3 and its corollaries). Rather unexpectedly, no range condition is assumed in Theorem 2.

All of our results have their roots in the following triple equality.

TheOREM 1. Let $D$ be a subset of a Banach space $X$ and $T: D \rightarrow X$ a firmly nonexpansive mapping. If $T$ can be iterated at $x \in D$, then for all $k \geqslant 1$,

$$
\lim _{n \rightarrow \infty}\left|T^{n+1} x-T^{n} x\right|=\lim _{n \rightarrow \infty}\left|T^{n+k} x-T^{n} x\right| / k=\lim _{n \rightarrow \infty}\left|T^{n} x / n\right| .
$$

Proof. Since $T$ is nonexpansive, it is clear that the limits

$$
L=\lim _{n \rightarrow \infty}\left|T^{n+1} x-T^{n} x\right| \text { and } R=\lim _{n \rightarrow \infty}\left|T^{n+k} x-T^{n} x\right|
$$

exist, and that $R \leqslant k L$. Also, the second equality follows from the first just as in [1, Theorem 2.1]. Therefore all we have to show is that $R \geqslant k L$. To this end we use induction on $k$. Since the case $k=1$ is clear, we assume that $R=j L$ for all

Received by the editors July 1, 1986.

1980 Mathematics Subject Classification (1985 Revision). Primary 47H09.

The first author was partially supported by the Fund for the Promotion of Research at the Technion. 
$1 \leqslant j \leqslant k$ and consider $R /(k+1)$. Given a positive $\varepsilon$ and letting $x_{n}=T^{n} x$, we can find an $N(\varepsilon)$ such that

$$
L-\varepsilon<\left|x_{n+j}-x_{n}\right| / j<L+\varepsilon
$$

for all $1 \leqslant j \leqslant k$ and $n>N(\varepsilon)$. Since $T$ is firmly nonexpansive, we also have

$$
\begin{aligned}
\left|x_{n+1}-x_{n+k+1}\right| & \leqslant\left|x_{n}+x_{n+1}-\left(x_{n+k}+x_{n+k+1}\right)\right| / 2 \\
& \leqslant\left|x_{n}-x_{n+k+1}\right| / 2+\left|x_{n+1}-x_{n+k}\right| / 2 .
\end{aligned}
$$

Hence

$$
\begin{aligned}
\left|x_{n}-x_{n+k+1}\right| & \geqslant 2\left|x_{n+1}-x_{n+k+1}\right|-\left|x_{n+1}-x_{n+k}\right| \\
& >2 k(L-\varepsilon)-(k-1)(L+\varepsilon)=(k+1) L-(3 k-1) \varepsilon,
\end{aligned}
$$

and the result follows.

Recall that a mapping $T: D \rightarrow X$ is said to be asymptotically regular [3] at $x \in D$ if $T$ can be iterated at $x$ and $\lim _{n \rightarrow \infty}\left(T^{n} x-T^{n+1} x\right)=0$. Our first corollary solves a problem which was left open in [5, p. 465].

COROllary 1. A firmly nonexpansive mapping which has a fixed point is asymptotically regular at each point where it can be iterated.

The next result identifies the common limit of Theorem 1.

Corollary 2. Let $T: D \rightarrow D$ be firmly nonexpansive, and set $d=\inf \{|y-T y|$ : $y \in D\}$. Then for each $x$ in $D, \lim _{n \rightarrow \infty}\left|T^{n+1} x-T^{n} x\right|=d$.

Recall that a closed convex subset $C$ of a Banach space has the fixed point property for nonexpansive mappings (FPP for short) if every nonexpansive $T$ : $C \rightarrow C$ has a fixed point.

Corollary 3. Let $C$ be a closed convex subset of a Banach space $X$ and $T: C \rightarrow C$ a firmly nonexpansive mapping. If each bounded closed convex subset of $X$ has the FPP, then $T$ is fixed point free if and only if $\lim _{n \rightarrow \infty}\left|T^{n} x\right|=\infty$ for all $x$ in $C$.

This result improves upon [5, Theorem 2.4(c)] because each bounded closed convex subset of a uniformly convex Banach space does indeed have the FPP. It cannot, however, be obtained by the approach of [5] because in general not every firmly nonexpansive mapping is strongly nonexpansive.

The next result is essentially known (cf. [7, p. 53]). It can now be viewed as a consequence of Corollary 1 and the nonlinear mean ergodic theorem.

COROLlary 4. Let $C$ be a closed convex subset of a uniformly convex Banach space with a Fréchet differentiable norm. If a firmly nonexpansive $T: C \rightarrow C$ has a fixed point, then for each $x$ in $C$ the sequence of iterates $\left\{T^{n} x\right\}$ converges weakly to a fixed point of $T$.

In order to continue we need a new geometric property of (infinite-dimensional) Banach spaces. First recall that the duality map $J$ from $X$ into the family of nonempty closed convex subsets of its dual $X^{*}$ is defined by

$$
J(x)=\left\{x^{*} \in X^{*}:\left(x, x^{*}\right)=|x|^{2}=\left|x^{*}\right|^{2}\right\} .
$$


To each functional $x^{*}$ in the unit sphere of $X^{*}$ there corresponds a face $F$ of the unit sphere $U$ of $X$, namely $J^{-1}\left(x^{*}\right)$. We shall say that the norm of $X$ is locally uniformly Fréchet differentiable (LUF) if for each face $F$ of $U$ the limit

$$
\lim _{t \rightarrow 0}(|x+t y|-|x|) / t
$$

is attained uniformly for all $y$ in $U$ and $x$ in $F$. It is clear that $X$ is LUF whenever $X^{*}$ is uniformly convex or $X$ has a Fréchet-differentiable norm and compact faces. It can also be shown that if $X$ is reflexive and locally uniformly convex, then $X^{*}$ is LUF.

We shall use the following fact.

Lemma 1. Let $\left\{x_{n}\right\}$ be a sequence in $X$ for which there exist $x \in X$ with $|x|=1$ and a sequence $x_{n}^{*} \subset J(x)$ such that $\lim _{n \rightarrow \infty}\left|x_{n}\right|=\lim _{n \rightarrow \infty}\left(x_{n}, x_{n}^{*}\right)$. If $X^{*}$ is LUF, then $\left\{x_{n}\right\}$ converges.

For reflexive $X$, the converse of Lemma 1 is also true.

Now let $D$ be a subset of a Banach space $X$ and $T: D \rightarrow X$ a nonexpansive mapping. If $T$ can be iterated at $x \in D$, then $L=\lim _{n \rightarrow \infty}\left|T^{n} x\right| / n$ exists. We shall also need the following lemma from [9].

Lemma 2. There exists a functional $z(x) \in X^{*}$ such that $|z(x)|=L$ and

$$
\left(\left(x-T^{m} x\right) / m, z(x)\right) \geqslant L^{2}
$$

for all $m \geqslant 1$.

Theorem 2. Let $D$ be a subset of a Banach space $X$ and $T: D \rightarrow X$ a firmly nonexpansive mapping. If $T$ can be iterated at $x \in D$ and $X^{*}$ is $L U F$, then the strong $\lim _{n \rightarrow \infty}\left(T^{n} x-T^{n+1} x\right)$ exists and is the unique point of least norm in the closure of the range of $I-T$.

Proof. Since the norm of $X^{*}$ is certainly Fréchet differentiable, we already know, by [9, Theorem 1], that $-v=\lim _{n \rightarrow \infty} T^{n} x / n$ exists. By Lemma 2 , for each $n \geqslant 0$ there exists a functional $z_{n} \in X^{*}$ such that $\left|z_{n}\right|=|v|$ and $\left(\left(T^{n} x-T^{n+m} x\right) / m, z_{n}\right)$ $\geqslant|v|^{2}$ for all $m \geqslant 1$. Letting $m \rightarrow \infty$ in this inequality we see that $z_{n} \in J(v)$ for all $n$. Setting $m=1$, we obtain $\left(T^{n} x-T^{n+1} x, z_{n}\right) \geqslant|v|^{2}$ for all $n$. Since

$$
\lim _{n \rightarrow \infty}\left|T^{n} x-T^{n+1} x\right|=|v|
$$

by Theorem 1 , we see that $\lim _{n \rightarrow \infty}\left(T^{n} x-T^{n+1} x, z_{n}\right)=|v|^{2}$, and

$$
v=\lim _{n \rightarrow \infty}\left(T^{n} x-T^{n+1} x\right)
$$

by Lemma 1. In order to identify $v$, let $d=\inf \{|y-T y|: y \in D\}$. It is clear that $|v| \geqslant d$. On the other hand, we have

$$
\left|T^{n} x-y\right| \leqslant\left|T^{n} x-y+r\left(T^{n} x-T^{n+1} x-(y-T y)\right)\right|
$$


for all $n \geqslant 0, y \in D$, and $r>0$ because $I-T$ is accretive. Dividing by $r=n / 2$ and then letting $n \rightarrow \infty$, we obtain

$$
2|v| \leqslant|v+(y-T y)| \leqslant|v|+|y-T y|
$$

for all $y \in D$. Hence $|v|=d$. If $u$ is another point of least norm in $\operatorname{cl}(R(I-T))$, then $|v+u|=|v|+|u|=2 d$, and $u=v$ because $X$ is strictly convex.

It may be of interest to observe that in this result $I-T$ is not assumed to satisfy any range condition.

It can be shown, however (cf. [9, Theorem 2]), that $I-T$ must satisfy the range condition at infinity introduced in [9].

As a matter of fact, Theorem 2 is valid for all those nonexpansive mappings which satisfy the conclusion of Theorem 1 . Consequently, we obtain considerable improvements not only of [5, Theorem 2.4(d)], but also of [1, Corollary 2.3 and 10, Corollary 5.3(b)] which deal with averaged and strongly nonexpansive mappings, respectively. The example of [8] can be used to show that the Fréchet differentiability of the norm of $X^{*}$ is a necessary condition for these results to hold. Although we do not know if it is sufficient, we can show that if $X^{*}$ is $(F)$ and $T$ satisfies the triple equality of Theorem 1, then there is a subsequence of $\left\{T^{n} x-T^{n+1} x\right\}$ which converges strongly. Moreover, all such subsequential limits coincide with the unique point of least norm in $\operatorname{cl}(R(I-T))$.

It is expected that related results for infinite products of resolvents and certain nonlinear semigroups, as well as weak convergence theorems, will appear elsewhere.

We conclude this note with a few remarks on the Hilbert ball $B$ equipped with the hyperbolic metric $\rho$ [7]. Without going into details, we recall that a mapping $T$ : $B \rightarrow B$ is firmly nonexpansive (of the first kind) if for each $x$ and $y$ in $B$, the function $g:[0,1] \rightarrow[0, \infty)$ defined by

$$
g(s)=\rho((1-s) x \oplus s T x,(1-s) y \oplus s T y)
$$

is nonincreasing.

THEOREM 3. Let $T$ be a firmly nonexpansive mapping in the Hilbert ball $B$. Then for each $x$ in $B$ and all $k \geqslant 1$,

$$
\lim _{n \rightarrow \infty} \rho\left(T^{n+1} x, T^{n} x\right)=\lim _{n \rightarrow \infty} \rho\left(T^{n+k} x, T^{n} x\right) / k=\lim _{n \rightarrow \infty} \rho\left(x, T^{n} x\right) / n .
$$

This result is proved by using the idea of the proof of Theorem 1 . We then use the methods of [11] to deduce the following corollaries. A different proof of Corollary 7 can be found on p. 140 of [7].

Corollary 5. Let $T: B \rightarrow B$ be firmly nonexpansive, and set $d=\inf \{\rho(y, T y)$ : $y \in B\}$. Then for each $x$ in $B, \lim _{n \rightarrow \infty} \rho\left(T^{n+1} x, T^{n} x\right)=d$.

COROLlaRy 6. Let $T$ be a firmly nonexpansive mapping in the Hilbert ball $B$. If $T$ is fixed point free, then for each $x$ in $B$ the sequence of iterates $\left\{T^{n} x\right\}$ converges strongly to $e(T)$, a point on the boundary of $B$. 
COROLlaRY 7. If a firmly nonexpansive $T: B \rightarrow B$ has a fixed point, then for each $x$ in $B$ the sequence of iterates $\left\{T^{n} x\right\}$ converges weakly to a fixed point of $T$.

Although we do not have an analogue of Theorem 3 for firmly holomorphic (or more generally, for firmly nonexpansive mappings of the second kind), Corollaries 6 and 7 are valid for such mappings (see $[6,7])$.

\section{REFERENCES}

1. J. B. Baillon, R. E. Bruck, and S. Reich, On the asymptotic behavior of nonexpansive mappings and semigroups in Banach spaces, Houston J. Math. 4 (1978), 1-9.

2. F. E. Browder, Convergence theorems for sequences of nonlinear operators in Banach spaces, Math. Z 100 (1967), 201-225.

3. F. E. Browder and W. V. Petryshyn, The solution by iteration of nonlinear functional equations in Banach spaces, Bull. Amer. Math. Soc. 72 (1966), 571-575.

4. R. E. Bruck, Nonexpansive projections on subsets of Banach spaces, Pacific J. Math. 47 (1973), $341-355$.

5. R. E. Bruck and S. Reich, Nonexpansive projections and resolvents of accretive operators in Banach spaces, Houston J. Math. 3 (1977), 459-470.

6. K. Goebel and S. Reich, Iterating holomorphic self-mappings of the Hilbert ball, Proc. Japan Acad. 58 (1982), 349-352.

7. Uniform convexity, hyperbolic geometry and nonexpansive mappings, Marcel Dekker, New York and Basel, 1984.

8. E. Kohlberg and A. Neyman, Asymptotic behavior of nonexpansive mappings in normed linear spaces, Israel J. Math. 38 (1981), 269-275.

9. A. T. Plant and S. Reich, The asymptotics of nonexpansive iterations, J. Funct. Anal. 54 (1983), 308-319.

10. S. Reich, On the asymptotic behavior of nonlinear semigroups and the range of accretive operators II, MRC Report \#2198, 1981; J. Math. Anal. Appl. 87 (1982), 134-146.

11. , Averaged mappings in the Hilbert ball, J. Math. Anal. Appl. 109 (1985), 199-206.

Department of Mathematics, University of Southern California, los Angeles, California 90089

Department of Mathematics, Technion-Israel Institute of Technology, Haifa 32000, Israel 